\title{
A note on the existence and uniqueness of the solutions to SFDES
}

Yeol JE Cho' ${ }^{1}$, Sever S Dragomir ${ }^{2}$ and Young-Ho Kim ${ }^{3 *}$

* Correspondence:

yhkim@changwon.ac.kr

${ }^{3}$ Department of Mathematics,

Changwon National University,

Changwon 641-773, Republic of

Korea

Full list of author information is available at the end of the article

\begin{abstract}
In this paper, we establish a new proof of the existence and uniqueness of solution to stochastic functional differential equations with infinite delay at phase space BC $\left((-\infty, 0]: R^{d}\right)$ which denotes the family of bounded continuous $R^{d}$-valued functions $\phi$ defined on $(-\infty, 0]$ with norm $\|\phi\|=\sup _{-\infty}<\theta \leq 0|\phi(\theta)|$.

Mathematics Subject Classification (2000): 60H05, 60H10.
\end{abstract}

Keywords: existence, uniqueness, stochastic functional differential equations, infinite delay.

\section{Introduction}

Stochastic differential equations (SDEs) are well known to model problems from many areas of science and engineering. For instance, in 2006, Henderson and Plaschko [1] published the SDEs in Science and Engineering, in 2007, Mao [2] published the SDEs, in 2010, Li and $\mathrm{Fu}$ [3] considered the stability analysis of stochastic functional differential equations with infinite delay and its application to recurrent neural networks.

In recent years, there is an increasing interest in stochastic functional differential equations(SFDEs) with finite and infinite delay under less restrictive conditions than Lipschitz condition. For instance, in 2007, Wei and Wang [4] discussed the existence and uniqueness of the solution for stochastic functional differential equations with infinite delay, in 2008, Mao et al. [5] discussed almost surely asymptotic stability of neutral stochastic differential delay equations with Markovian switching, in 2008, Ren et al. [6] considered the existence and uniqueness of the solutions to SFDEs with infinite delay, and in 2009, Ren and Xia [7], discussed the existence and uniqueness of the solution to neutral SFDEs with infinite delay. Furthermore, on this topic, one can see Halidias [8], Henderson and Plaschko [1], Kim [9,10], Ren [11], Ren and Xia [7], Taniguchi [12] and references therein for details.

On the other hand, Mao [2] discussed $d$-dimensional stochastic functional differential equations with finite delay

$$
\mathrm{d} x(t)=f\left(x_{t}, t\right) \mathrm{d} t+g\left(x_{t}, t\right) \mathrm{d} B(t), \quad t_{0} \leq t \leq T,
$$

where $x_{t}=\{x(t+\theta):-\tau \leq \theta \leq 0\}$ could be considered as a $C\left([-\tau, 0] ; R^{d}\right)$-value stochastic process. The initial value of (1.1) was proposed as follows: 


$$
\begin{aligned}
& x_{t_{0}}=\xi=\{\xi(\theta):-\tau \leq \theta \leq 0\} \quad \text { is an } \mathcal{F}_{t_{0}} \text { - measurable } \\
& C\left([-\tau, 0] ; R^{d}\right) \text { - value random variable such that } E\|\xi\|^{2}<\infty .
\end{aligned}
$$

Furthermore, Ren et al. [6] also considered the stochastic functional differential equations with infinite delay at phase space $\mathrm{BC}\left((-\infty, 0] ; R^{d}\right)$ to be described below.

$$
\mathrm{d} x(t)=f\left(x_{t}, t\right) \mathrm{d} t+g\left(x_{t}, t\right) \mathrm{d} B(t), \quad t_{0} \leq t \leq T,
$$

where $x_{t}=\{X(t+\theta):-\infty \leq \theta \leq 0\}$ could be considered as a $\mathrm{BC}\left((-\infty, 0] ; R^{d}\right)$-value stochastic process. The initial value of (1.2) was proposed as follows:

$$
\begin{aligned}
& X_{t_{0}}=\xi=\{\xi(\theta):-\infty \leq \theta \leq 0\} \quad \text { is an } \mathcal{F}_{t_{0}} \text { - measurable } \\
& \operatorname{BC}\left((-\infty, 0] ; R^{d}\right) \text { - value random variable such that } \xi \in \mathcal{M}^{2}\left((-\infty, 0] ; R^{d}\right) .
\end{aligned}
$$

Following this way, now we recall the existence and uniqueness of the solutions to the Equation 1.2 with initial data (1.3) under the non-Lipschitz condition and the weakened linear growth condition. In this paper, we will give some new proof of the existence and uniqueness of the solutions to ISDEs under an alternative way.

\section{Preliminary}

Let $|\cdot|$ denote Euclidean norm in $R^{n}$. If $A$ is a vector or a matrix, its transpose is denoted by $A^{T}$; if $A$ is a matrix, its trace norm is represented by $|A|=\sqrt{\operatorname{trace}\left(A^{T} A\right)}$. Let $t_{0}$ be a positive constant and $(\Omega, \mathcal{F}, P)$, throughout this paper unless otherwise specified, be a complete probability space with a filtration $\left\{\mathcal{F}_{t}\right\}_{t \geq t_{0}}$ satisfying the usual conditions (i.e. it is right continuous and $\mathcal{F}_{t_{0}}$ contains all $P$-null sets). Assume that $B(t)$ is a $m$ dimensional Brownian motion defined on complete probability space, that is $B(t)=\left(B_{1}\right.$ $\left.(t), B_{2}(t), \ldots, B_{m}(t)\right)^{T}$. Let $\mathrm{BC}\left((-\infty, 0] ; R^{d}\right)$ denote the family of bounded continuous $R^{d}$ value functions $\phi$ defined on $(-\infty, 0]$ with norm $\|\phi\|=\sup _{-\infty}<\theta \leq 0|\phi(\theta)|$. We denote by $\mathcal{M}^{2}\left((-\infty, 0] ; R^{d}\right)$ the family of all $\mathcal{F}_{t_{0}}$-measurable, $\mathbb{R}^{d}$-valued process $\psi(t)=\psi(t, w), t \in$ $(-\infty, 0]$, such that $E \int_{-\infty}^{0}|\psi(t)|^{2} \mathrm{~d} t<\infty$. And let $\mathcal{L}^{p}\left([a, b] ; R^{d}\right)$ is the family of $R^{d}$-valued $\mathcal{F}_{t}$-adapted processes $\{f(t)\}_{a \leq t \leq b}$ such that $\int_{a}^{b}|f(t)|^{p} \mathrm{~d} t<\infty$.

With all the above preparation, consider a $d$-dimensional stochastic functional differential equations:

$$
\mathrm{d} x(t)=f\left(x_{t}, t\right) \mathrm{d} t+g\left(x_{t}, t\right) \mathrm{d} B(t), \quad t_{0} \leq t \leq T,
$$

where $x_{t}=\{x(t+\theta):-\infty<\theta \leq 0\}$ can be considered as a $\mathrm{BC}\left((-\infty, 0] ; R^{d}\right)$-value stochastic process, where

$$
\begin{aligned}
& f: \mathrm{BC}\left((-\infty, 0] ; R^{d}\right) \times\left[t_{0}, T\right] \rightarrow R^{d}, \\
& g: \mathrm{BC}\left((-\infty, 0] ; R^{d}\right) \times\left[t_{0}, T\right] \rightarrow R^{d \times m}
\end{aligned}
$$

be Borel measurable. Next, we give the initial value of (2.1) as follows:

$$
\begin{aligned}
& x_{t_{0}}=\xi=\{\xi(\theta):-\infty<\theta \leq 0\} \text { is an } \mathcal{F}_{t_{0}} \text { - measurable } \\
& \operatorname{BC}\left((-\infty, 0] ; R^{d}\right) \text { - value random variable such that } \xi \in \mathcal{M}^{2}\left((-\infty, 0] ; R^{d}\right) .
\end{aligned}
$$


To find out the solution, we give the definition of the solution of the Equation 2.1 with initial data (2.2).

Definition 2.1. [6] $R^{d}$-value stochastic process $x(t)$ defined on $-\infty<t \leq T$ is called the solution of (2.1) with initial data (2.2), if $x(t)$ has the following properties:

(i) $x(t)$ is continuous and $\{x(t)\}_{t_{0} \leq t \leq T}$ is $\mathcal{F}_{t}$-adapted;

(ii) $\left\{f\left(x_{t}, t\right)\right\} \in \mathcal{L}^{1}\left(\left[t_{0}, T\right] ; R^{d}\right)$ and $\left\{g\left(x_{t}, t\right)\right\} \in \mathcal{L}^{2}\left(\left[t_{0}, T\right] ; R^{d^{\times m}}\right)$;

(iii) $x_{t_{0}}=\xi$, for each $t_{0} \leq t \leq T$,

$$
x(t)=\xi(0)+\int_{t_{0}}^{t} f\left(x_{s}, s\right) \mathrm{d} s+\int_{t_{0}}^{t} g\left(x_{s}, s\right) \mathrm{d} B(s) \quad \text { a.s. }
$$

A solution $x(t)$ is called as a unique if any other solution $\bar{x}(t)$ is indistinguishable with $x(t)$, that is

$$
P\{x(t)=\bar{x}(t), \text { for any }-\infty<t \leq T\}=1 .
$$

The integral inequalities of Gronwall type have been applied in the theory of SDEs to prove the results on existence, uniqueness, and stability etc. [10,13-16]. Naturally, Gronwall's inequality will play an important role in next section.

Lemma 2.1. (Gronwall's inequality). Let $u(t)$ and $b(t)$ be non-negative continuous functions for $t \geq \alpha$, and let

$$
u(t) \leq a+\int_{\alpha}^{t} b(s) u(s) \mathrm{d} s, \quad t \geq \alpha
$$

where $a \geq 0$ is a constant. Then

$$
u(t) \leq a \exp \left(\int_{\alpha}^{t} b(s) \mathrm{d} s\right), \quad t \geq \alpha
$$

Lemma 2.2. (Bihari's inequality). Let $u$ and $b$ be non-negative continuous functions defined on $R_{+}$. Let $g(u)$ be a non-decreasing continuous function on $R_{+}$and $g(u)>0$ on $(0, \infty)$. If

$$
u(t) \leq k+\int_{0}^{t} b(s) g(u(s)) \mathrm{d} s,
$$

for $t \in R_{+}$, where $k \geq 0$ is a constant. Then for $0 \leq t \leq t_{1}$,

$$
u(t) \leq G^{-1}\left(G(k)+\int_{0}^{t} b(s) \mathrm{d} s\right)
$$

where

$$
G(r)=\int_{r_{0}}^{r} \frac{\mathrm{d} s}{g(s)}, \quad r>0, \quad r_{0}>0,
$$


and $G^{-1}$ is the inverse function of $G$ and $t_{1} \in R_{+}$is chosen so that

$$
G(k)+\int_{0}^{t} b(s) \mathrm{d} s \in \operatorname{Dom}\left(G^{-1}\right)
$$

for all $t \in R_{+}$lying in the interval $0 \leq t \leq t_{1}$.

The following two lemmas are known as the moment inequality for stochastic integrals which will play an important role in next section.

Lemma 2.3. [2]. If $p \geq 2, g \in \mathcal{M}^{2}\left([0, T] ; R^{d \times m}\right)$ such that

$$
E \int_{0}^{T}|g(s)|^{p} \mathrm{~d} s<\infty
$$

then

$$
E\left|\int_{0}^{T} g(s) \mathrm{d} B(s)\right|^{p} \leq\left(\frac{p(p-1)}{2}\right)^{\frac{p}{2}} T^{\frac{p-2}{2}} E \int_{0}^{T}|g(s)|^{p} \mathrm{~d} s .
$$

In particular, for $p=2$, there is equality.

Lemma 2.4. [2]. If $p \geq 2, g \in \mathcal{M}^{2}\left([0, T] ; R^{d \times m}\right)$ such that

$$
E \int_{0}^{T}|g(s)|^{p} \mathrm{~d} s<\infty
$$

then

$$
E\left(\sup _{0 \leq t \leq T}\left|\int_{0}^{t} g(s) \mathrm{d} B(s)\right|^{p}\right) \leq\left(\frac{p^{3}}{2(p-1)}\right)^{\frac{p}{2}} T^{\frac{p-2}{2}} E \int_{0}^{T}|g(s)|^{p} \mathrm{~d} s .
$$

\section{Existence and Uniqueness of the Solutions}

In order to obtain the existence and uniqueness of the solutions to (2.1) with initial data (2.2), we define $x_{t_{0}}^{0}=\xi$ and $x^{0}(t)=\xi(0)$, for $t_{0} \leq t \leq T$. Let $x_{t_{0}}^{n}=\xi, n=1,2, \ldots$ and define the Picard sequence

$$
x^{n}(t)=\xi(0)+\int_{t_{0}}^{t} f\left(x_{s}^{n-1}, s\right) \mathrm{d} s+\int_{t_{0}}^{t} g\left(x_{s}^{n-1}, s\right) \mathrm{d} B(s), \quad t_{0} \leq t \leq T .
$$

Now we begin to establish the theory of the existence and uniqueness of the solution. We first show that the non-Lipschitz condition and the weakened linea growth condition guarantee the existence and uniqueness.

Theorem 3.1. Assume that there exist a positive number $K$ such that

(i) For any $\phi, \psi \in \mathrm{BC}\left((-\infty, 0] ; R^{d}\right)$ and $t \in\left[t_{0}, T\right]$, it follows that

$$
|f(\varphi, t)-f(\psi, t)|^{2} \vee|g(\varphi, t)-g(\psi, t)|^{2} \leq \kappa\left(\|\varphi-\psi\|^{2}\right),
$$


where $\kappa(\cdot)$ is a concave non-decreasing function from $\mathbb{R}_{+}$to $\mathbb{R}_{+}$such that $\kappa(0)=0$, $\kappa(u)>0$ for $u>0$ and $\int_{0^{+}} \mathrm{d} u / \kappa(u)=\infty$.

(ii) For any $t \in\left[t_{0}, T\right]$, it follows that $f(0, t), g(0, t) \in L^{2}$ such that

$$
|f(0, t)|^{2} \vee|g(0, t)|^{2} \leq K .
$$

Then the initial value problem (2.1) has a solution $x(t)$. Moreover, $x(t) \in \mathcal{M}^{2}\left((-\infty, T] ; R^{d}\right)$. We prepare a lemma to prove this theorem.

Lemma 3.2. Let the assumption (3.1) and (3.2) of Theorem 3.1 hold. If $x(t)$ is a solution of equation (2.1) with initial data (2.2), then

$$
E\left(\sup _{-\infty<t \leq T}|x(t)|^{2}\right) \leq E\|\xi\|^{2}+c_{2} e^{6 b\left(T-t_{0}+1\right)\left(T-t_{0}\right)},
$$

where $c_{2}=c_{1}+E \bigotimes \xi \nabla^{2}, c_{1}=3 E \nabla \xi \nabla^{2}+6\left(T-t_{0}+1\right)\left(T-t_{0}\right)[K+a]$. In particular, $x$ (t) belong to $\mathcal{M}^{2}\left((-\infty, T] ; R^{d}\right)$.

Proof. For each number $n \geq 1$, define the stopping time

$$
\tau_{n}=T \wedge \inf \left\{t \in\left[t_{0}, T\right]:\|x(t)\| \geq n\right\} .
$$

Obviously, as $n \rightarrow \infty, \tau_{n} \uparrow T$ a.s. Let $x^{n}(t)=x\left(t \wedge \tau_{n}\right), t \in(-\infty, T]$. Then, for $t_{0} \leq t \leq$ $T, x^{n}(t)$ satisfy the following equation

$$
\left.x^{n}(t)=\xi(0)+\int_{t_{0}}^{t} f\left(x_{s}^{n}, s\right)\right) I_{\left[t_{0}, \tau_{n}\right]}(s) \mathrm{d} s+\int_{t_{0}}^{t} g\left(x_{s}^{n}, s\right) I_{\left[t_{0}, \tau_{n}\right]}(s) \mathrm{d} B(s) .
$$

Using the elementary inequality $\left(\sum x_{i}\right)^{p} \leq n^{p-1} \sum x_{i}^{p}$ when $p \geq 1$, we have

$$
\left|x^{n}(t)\right|^{2} \leq 3|\xi(0)|^{2}+3\left|\int_{t_{0}}^{t} f\left(x_{s}^{n}, s\right) I_{\left[t_{0}, \tau_{n}\right]}(s) \mathrm{d} s\right|^{2}+3\left|\int_{t_{0}}^{t} g\left(x_{s}^{n}, s\right) I_{\left[t_{0}, \tau_{n}\right]}(s) \mathrm{d} B(s)\right|^{2} .
$$

By the Hölder's inequality and the moment inequality, we have

$$
E\left|x^{n}(t)\right|^{2} \leq 3\left(E\|\xi\|^{2}+\left(t-t_{0}\right) E \int_{t_{0}}^{t}\left|f\left(x_{s}^{n}, s\right)\right|^{2} \mathrm{~d} s+E \int_{t_{0}}^{t}\left|g\left(x_{s}^{n}, s\right)\right|^{2} \mathrm{~d} s\right) .
$$

Hence, by the condition (3.1) and (3.2) one can further show that

$$
E\left|x^{n}(t)\right|^{2} \leq 3 E\|\xi\|^{2}+6\left(t-t_{0}+1\right)\left(E \int_{t_{0}}^{t} \kappa\left(\left\|x_{s}^{n}\right\|^{2}\right) \mathrm{d} s+E \int_{t_{0}}^{t} K \mathrm{~d} s\right) .
$$

Given that $\kappa(\cdot)$ is concave and $\kappa(0)=0$, we can find a positive constants $a$ and $b$ such that $\kappa(u) \leq a+b u$ for $u \geq 0$. So, we obtains that

$$
E\left(\sup _{t_{0} \leq s \leq t}\left|x^{n}(s)\right|^{2}\right) \leq c_{1}+6 b\left(t-t_{0}+1\right) \int_{t_{0}}^{t} E\left\|x_{s}^{n}\right\|^{2} \mathrm{~d} s
$$

where $c_{1}=3 E\|\xi\|^{2}+6\left(T-t_{0}+1\right)\left(T-t_{0}\right)[K+a]$. Noting the fact that $\sup _{-\infty<s \leq t}\left|x^{n}(s)\right|^{2} \leq\|\xi\|^{2}+\sup _{t_{0} \leq s \leq t}\left|x^{n}(s)\right|^{2}$, we obtain 


$$
E\left(\sup _{-\infty<s \leq t}\left|x^{n}(s)\right|^{2}\right) \leq c_{2}+6 b\left(t-t_{0}+1\right) \int_{t_{0}}^{t} E\left(\sup _{-\infty<r \leq s}\left|x^{n}(r)\right|^{2}\right) \mathrm{d} s,
$$

where $c_{2}=c_{1}+E|| \xi \|^{2}$. So, by the Gronwall inequality yields that

$$
E\left(\sup _{-\infty<s \leq t}\left|x^{n}(s)\right|^{2}\right) \leq c_{2} \exp \left(6 b\left(T-t_{0}+1\right)\left(T-t_{0}\right)\right) .
$$

Letting $t=T$, it then follows that

$$
E\left(\sup _{-\infty<s \leq T}\left|x\left(s \wedge \tau_{n}\right)\right|^{2}\right) \leq E\|\xi\|^{2}+c_{2} e^{6 b\left(T-t_{0}+1\right)\left(T-t_{0}\right)} .
$$

Thus

$$
E\left(\sup _{-\infty<s \leq \tau_{n}}|x(s)|^{2}\right) \leq E\|\xi\|^{2}+c_{2} e^{6 b\left(T-t_{0}+1\right)\left(T-t_{0}\right)} .
$$

Consequently the required result follows by letting $n \rightarrow \infty$. $\square$

Proof of Theorem 3.1. Let $x(t)$ and $\bar{x}(t)$ be any tow solutions of (2.1). By Lemma 3.2, $x(t), \bar{x}(t) \in \mathcal{M}^{2}\left((-\infty, T] ; R^{d}\right)$. Note that

$$
x(t)-\bar{x}(t)=\int_{t_{0}}^{t}\left[f\left(x_{s}, s\right)-f\left(\bar{x}_{s}, s\right)\right] \mathrm{d} s+\int_{t_{0}}^{t}\left[g\left(x_{s}, s\right)-g\left(\bar{x}_{s}, s\right)\right] \mathrm{d} B(s) .
$$

By the elementary inequality $(u+v)^{2} \leq 2\left(u^{2}+v^{2}\right)$, one then gets

$$
|x(t)-\bar{x}(t)|^{2}=2\left|\int_{t_{0}}^{t}\left[f\left(x_{s}, s\right)-f\left(\bar{x}_{s}, s\right)\right] \mathrm{d} s\right|^{2}+2\left|\int_{t_{0}}^{t}\left[g\left(x_{s}, s\right)-g\left(\bar{x}_{s}, s\right)\right] \mathrm{d} B(s)\right|^{2} .
$$

By the Hölder's inequality, the moment inequality, and (3.1) we have

$$
E\left(\sup _{t_{0} \leq s \leq t}|x(s)-\bar{x}(s)|^{2}\right) \leq 2\left(T-t_{0}+1\right) E \int_{t_{0}}^{t} \kappa\left(\left\|x_{s}-\bar{x}_{s}\right\|^{2}\right) \mathrm{d} s .
$$

Since $\kappa(\cdot)$ is concave, by the Jensen inequality, we have

$$
E \kappa\left(\left\|x_{s}-\bar{x}_{s}\right\|^{2}\right) \leq \kappa\left(E\left\|x_{s}-\bar{x}_{s}\right\|^{2}\right) .
$$

Consequently, for any $\epsilon>0$,

$$
E\left(\sup _{t_{0} \leq s \leq t}|x(s)-\bar{x}(s)|^{2}\right) \leq \epsilon+2\left(T-t_{0}+1\right) \int_{t_{0}}^{t} \kappa\left[E\left(\sup _{t_{0} \leq r \leq s}|x(r)-\bar{x}(r)|^{2}\right)\right] \mathrm{d} s .
$$

By the Bihari inequality, one deduces that, for all sufficiently small $\epsilon>0$,

$$
E\left(\sup _{t_{0} \leq s \leq t}|x(s)-\bar{x}(s)|^{2}\right) \leq G^{-1}\left[G(\epsilon)+2\left(T-t_{0}+1\right)\left(T-t_{0}\right)\right],
$$


where

$$
G(r)=\int_{1}^{r} \frac{1}{\kappa(u)} \mathrm{d} u
$$

on $r>0$, and $G^{-1}(\cdot)$ be the inverse function of $G(\cdot)$. By assumption $\int_{0^{+}} \frac{\mathrm{d} u}{\kappa(u)}=\infty$ and the definition of $\kappa(\cdot)$, one sees that $\lim _{\epsilon \downarrow 0} G(\epsilon)=-\infty$ and then

$$
\lim _{\epsilon \downarrow 0} G^{-1}\left[G(\epsilon)+2\left(T-t_{0}+1\right)\left(T-t_{0}\right)\right]=0 .
$$

Therefore, by letting $\epsilon \rightarrow 0$ in (3.3), gives

$$
E\left(\sup _{t_{0} \leq t \leq T}|x(t)-\bar{x}(t)|^{2}\right)=0 .
$$

This implies that $x(t)=\bar{x}(t)$ for $t_{0} \leq t \leq T$, Therefore, for all $-\infty<t \leq T, x(t)=\bar{x}(t)$ a. s. The uniqueness has been proved.

Next to check the existence. Define $x_{t_{0}}^{0}=\xi$ and $x^{0}(t)=\xi(0)$ for $t_{0} \leq t \leq T$. For each $n$ $=1,2, \ldots$, set $x_{t_{0}}^{n}=\xi$ and define, by the Picard iterations,

$$
x^{n}(t)=\xi(0)+\int_{t_{0}}^{t} f\left(x_{s}^{n-1}, s\right) \mathrm{d} s+\int_{t_{0}}^{t} g\left(x_{s}^{n-1}, s\right) \mathrm{d} B(s)
$$

for $t_{0} \leq t \leq T$. Obviously, $x^{0}(t) \in \mathcal{M}^{2}\left(\left[t_{0}, T\right]: R^{d}\right)$. Moreover, it is easy to see that $x^{n}(t) \in \mathcal{M}^{2}\left((-\infty, T]: R^{d}\right)$, in fact

$$
\left|x^{n}(t)\right|^{2} \leq 3|\xi(0)|^{2}+3\left|\int_{t_{0}}^{t} f\left(x_{s}^{n-1}, s\right) \mathrm{d} s\right|^{2}+3\left|\int_{t_{0}}^{t} g\left(x_{s}^{n-1}, s\right) \mathrm{d} B(s)\right|^{2} .
$$

Taking the expectation on both sides and using the Hölder inequality and moment inequality, we have

$$
\begin{aligned}
& E\left|x^{n}(t)\right|^{2} \\
& \leq 3 E\|\xi\|^{2}+3\left(t-t_{0}\right) E \int_{t_{0}}^{t}\left|f\left(x_{s}^{n-1}, s\right)\right|^{2} \mathrm{~d} s+3 E\left|\int_{t_{0}}^{t} g\left(x_{s}^{n-1}, s\right) \mathrm{d} B(s)\right|^{2} \\
& \leq 3 E\|\xi\|^{2}+3\left(t-t_{0}\right) E \int_{t_{0}}^{t}\left|f\left(x_{s}^{n-1}, s\right)-f(0, s)+f(0, s)\right|^{2} \mathrm{~d} s \\
& \quad+3 E \int_{t_{0}}^{t}\left|g\left(x_{s}^{n-1}, s\right)-g(0, s)+g(0, s)\right|^{2} \mathrm{~d} s .
\end{aligned}
$$


Using the elementary inequality $(u+v)^{2} \leq 2 u^{2}+2 v^{2}$, (3.1), and (3.2), we have

$$
\begin{aligned}
& E\left|x^{n}(t)\right|^{2} \\
& \quad \leq 3 E\|\xi\|^{2}+3\left(t-t_{0}+1\right) E \int_{t_{0}}^{t}\left[2 \kappa\left(\left\|x_{s}^{n-1}\right\|^{2}\right)+2 K\right] \mathrm{d} s \\
& \quad \leq 3 E\|\xi\|^{2}+6 K\left(T-t_{0}\right)\left(T-t_{0}+1\right)+6\left(T-t_{0}+1\right) E \int_{t_{0}}^{t} \kappa\left(\left\|x_{s}^{n-1}\right\|^{2}\right) \mathrm{d} s .
\end{aligned}
$$

Given that $\kappa(\cdot)$ is concave and $\kappa(0)=0$, we can find a positive constants $a$ and $b$ such that $\kappa(u) \leq a+b u$ for $u \geq 0$. So, we have

$$
E\left|x^{n}(t)\right|^{2} \leq c_{1}+6 b\left(T-t_{0}+1\right) \int_{t_{0}}^{t} E\left\|x_{s}^{n-1}\right\|^{2} \mathrm{~d} s
$$

where $c_{1}=\left.3 E|| \xi\right|^{2}+6\left(T-t_{0}\right)\left(T-t_{0}+1\right)[K+a]$. It also follows from the inequality that for any $k \geq 1$,

$$
\begin{aligned}
\max _{1 \leq n \leq k} E\left|x^{n}(t)\right|^{2} & \leq c_{1}+6 b\left(T-t_{0}+1\right) \int_{t_{0}}^{t} \max _{1 \leq n \leq k} E\left\|x^{n-1}(s)\right\|^{2} \mathrm{~d} s \\
& \leq c_{1}+6 b\left(T-t_{0}+1\right) \int_{t_{0}}^{t}\left(E\|\xi\|^{2}+\max _{1 \leq n \leq k} E\left|x^{n}(s)\right|^{2}\right) \mathrm{d} s \\
& \leq c_{2}+6 b\left(T-t_{0}+1\right) \int_{t_{0}}^{t} \max _{1 \leq n \leq k} E\left|x^{n}(s)\right|^{2} \mathrm{~d} s
\end{aligned}
$$

where $c_{2}=c_{1}=6 b\left(T-t_{0}\right)\left(T-t_{0}+1\right) E|| \xi \|^{2}$. The Gronwall inequality implies

$$
\max _{1 \leq n \leq k} E\left|x^{n}(t)\right|^{2} \leq c_{2} \exp \left(6 b\left(T-t_{0}\right)\left(T-t_{0}+1\right)\right) .
$$

Since $k$ is arbitrary, we must have

$$
E\left|x^{n}(t)\right|^{2} \leq c_{2} \exp \left(6 b\left(T-t_{0}+1\right)\left(T-t_{0}\right)\right)
$$

for all $t_{0} \leq t \leq T, n \geq 1$.

Next, we that the sequence $\left\{x^{n}(t)\right\}$ is Cauchy sequence. For all $n \geq 0$ and $t_{0} \leq t \leq T$, we have

$$
\begin{aligned}
& x^{n}(t)-x^{m}(t) \\
& \quad=\int_{t_{0}}^{t}\left[f\left(x_{s}^{n-1}, s\right)-f\left(x_{s}^{m-1}, s\right)\right] \mathrm{d} s+\int_{t_{0}}^{t}\left[g\left(x_{s}^{n-1}, s\right)-g\left(x_{s}^{m-1}, s\right)\right] \mathrm{d} B(s) .
\end{aligned}
$$

Next, using an elementary inequality $(u+v)^{2} \leq \frac{1}{\alpha} u^{2}+\frac{1}{1-\alpha} v^{2}$ and the condition (H3), we derive that 


$$
\begin{aligned}
& \left|x^{n}(t)-x^{m}(t)\right|^{2} \\
& \quad \leq \frac{1}{\alpha}\left|\int_{t_{0}}^{t}\left[f\left(x_{s}^{n-1}, s\right)-f\left(x_{s}^{m-1}, s\right)\right] \mathrm{d} s\right|^{2}+\frac{1}{1-\alpha}\left|\int_{t_{0}}^{t}\left[g\left(x_{s}^{n-1}, s\right)-g\left(x_{s}^{m-1}, s\right)\right] \mathrm{d} B(s)\right|^{2} .
\end{aligned}
$$

On the other hand, by Hölder's inequality, Lemma (2.4), and the condition, one can show that

$$
E\left(\sup _{t_{0}<s \leq t}\left|x^{n}(s)-x^{m}(s)\right|^{2}\right) \leq \beta \int_{t_{0}}^{t} \kappa\left(E\left(\sup _{t_{0} \leq u \leq s}\left|x^{n-1}(u)-x^{m-1}(u)\right|^{2}\right)\right) \mathrm{d} s,
$$

where $\beta=\left(T-t_{0}\right) / \alpha+4 /(1-\alpha)$. Let

$$
Z(t)=\lim _{n, m \rightarrow \infty} \sup E\left(\sup _{t_{0} \leq s \leq t}\left|x^{n}(s)-x^{m}(s)\right|^{2}\right) .
$$

From (3.6), for any $\epsilon>0$, we get

$$
Z(t) \leq \epsilon+\beta \int_{t_{0}}^{t} \kappa(Z(s)) \mathrm{d} s
$$

By the Bihari inequality, one deduces that, for all sufficiently small $\epsilon>0$,

$$
Z(t) \leq G^{-1}\left[G(\epsilon)+\beta\left(T-t_{0}\right)\right]
$$

where

$$
G(r)=\int_{1}^{r} \frac{1}{\kappa(u)} \mathrm{d} u
$$

on $r>0$, and $G^{-1}(\cdot)$ be the inverse function of $G(\cdot)$. By assumption, we get $Z(t)=0$. This shows the sequence $\left\{x^{n}(t), n \geq 0\right\}$ is a Cauchy sequence in $L^{2}$. Hence, as $n \rightarrow \infty$, $x^{n}(t)-x(t)$, that is $E\left|x^{n}(t)-x(t)\right|^{2} \rightarrow 0$. Letting $n \rightarrow \infty$ in (3.5) then yields that

$$
E\left(\sup _{t_{0} \leq s \leq t}|x(s)|^{2}\right) \leq c_{2} \exp \left(6 b\left(T-t_{0}+1\right)\left(T-t_{0}\right)\right)
$$

for all $t_{0} \leq t \leq T$. Therefore, $x(t) \in \mathcal{M}^{2}\left((-\infty, T] ; R^{d}\right)$. It remains to show that $x(t)$ satisfies Equation 2.3. Note that

$$
\begin{aligned}
& E\left|\int_{t_{0}}^{t}\left(f\left(x_{s}^{n}, s\right)-f\left(x_{s}, s\right)\right) \mathrm{d} s\right|^{2}+E\left|\int_{t_{0}}^{t}\left(g\left(x_{s}^{n}, s\right)-g\left(x_{s}, s\right)\right) \mathrm{d} B(s)\right|^{2} \\
& \quad \leq\left(t-t_{0}\right) E \int_{t_{0}}^{t}\left|\left(f\left(x_{s}^{n}, s\right)-f\left(x_{s}, s\right)\right)\right|^{2} \mathrm{~d} s+E \int_{t_{0}}^{t}\left|\left(g\left(x_{s}^{n}, s\right)-g\left(x_{s}, s\right)\right)\right|^{2} \mathrm{~d} s \\
& \quad \leq\left(t-t_{0}+1\right) \int_{t_{0}}^{T} \kappa\left(E\left(\sup _{t_{0} \leq u \leq s}\left|x^{n}(u)-x(u)\right|^{2}\right)\right) \mathrm{d} s .
\end{aligned}
$$


Noting that sequence $x^{n}(t)$ is uniformly converge on $(-\infty, T]$, it means that

$$
E\left(\sup _{t_{0} \leq u \leq s}\left|x^{n}(u)-x(u)\right|^{2}\right) \rightarrow 0
$$

as $n \rightarrow \infty$, further

$$
\kappa\left(E\left(\sup _{t_{0} \leq u \leq s}\left|x^{n}(u)-x(u)\right|^{2}\right)\right) \rightarrow 0
$$

as $n \rightarrow \infty$. Hence, taking limits on both sides in the Picard sequence, we obtain that

$$
x(t)=x_{0}+\int_{t_{0}}^{t} f\left(x_{s}, s\right) \mathrm{d} s+\int_{t_{0}}^{t} g\left(x_{s}, s\right) \mathrm{d} B(s)
$$

on $t_{0} \leq t \leq T$. The above expression demonstrates that $x(t)$ is the solution of (2.3). So, the existence of theorem is complete.

Remark 3.1. In the proof of Theorem 3.1, the solution is constructed by the successive approximation. It shows that how to get the approximate solution of (2.1) and how to construct Picard sequence $x^{n}(t)$. For SFDEs, we know that a weakened linear growth condition imposed on Theorem 3.1 is rigorous for our discussion. In papers $[6,7]$, the proofs of the assertions are based on some function inequalities. Recall that the procedures has become more and more complicated in those proofs. For this reason, although analogous problem is studied here, the proof of the assertion in the Theorem is completely different with respect to the ones from [7]. Moreover, our new proof in this paper is completed by Bihari's inequality and more simple than that reported in [7].

\section{Acknowledgements}

The authors wish to thank the anonymous referees for their endeavors and valuable comments. Also, this research was supported by Basic Science Research Program through the National Research Foundation of Korea(NRF) funded by the Ministry of Education, Science and Technology(2011-0023547)

\section{Author details}

${ }^{1}$ Department of Mathematics Education, Gyeongsang National University, Chinju 660-701, Republic of Korea ${ }^{2}$ School of Computer Science and Mathematics, Victoria University of Technology, P.O. Box 14428, MCMC Melbourne, VIC 8001, Australia ${ }^{3}$ Department of Mathematics, Changwon National University, Changwon 641-773, Republic of Korea

\section{Authors' contributions}

All authors read and approved the final manuscript.

\section{Competing interests}

The authors declare that they have no competing interests.

Received: 26 March 2012 Accepted: 7 June 2012 Published: 7 June 2012

\section{References}

1. Henderson, D, Plaschko, P: Stochastic Differential Equations in Science and Engineering. World Scientific Publishing Co., Singapore. (2006)

2. Mao, X: Stochastic Differential Equations and Applications. Horwood Publication, Chichester. (2007)

3. Li, X, Fu, X: Stability analysis of stochastic functional differential equations with infinite delay and its application to recurrent neural networks. J Comput Appl Math. 234, 407-417 (2010). doi:10.1016/j.cam.2009.12.033

4. Wei, F, Wang, K: The existence and uniqueness of the solution for stochastic functional differential equations with infinite delay. J Math Anal Appl. 331, 516-531 (2007). doi:10.1016/j.jmaa.2006.09.020

5. Mao, X, Shen, Y, Yuan, C: Almost surely asymptotic stability of neutral stochastic differential delay equations with Markovian switching. Stoch Process Appl. 118, 1385-1406 (2008). doi:10.1016/j.spa.2007.09.005

6. Ren, Y, Lu, S, Xia, N: Remarks on the existence and uniqueness of the solution to stochastic functional differential equations with infinite delay. J Comput Appl Math. 220, 364-372 (2008). doi:10.1016/j.cam.2007.08.022 
7. Ren, $Y, X i a, N$ : Existence, uniqueness and stability of the solution to neutral stochastic functional differential equations with infinite delay. Appl Math Comput. 210, 72-79 (2009). doi:10.1016/j.amc.2008.11.009

8. Halidias, N: Remarks and corrections on "An esistence theorem for stochastic functional differential equations with dealys under weak assumptions, Statistics and Probability Letters 78, 2008" by N. Halidias and Y. Ren. Stoch Probab Lett. 79, 2220-2222 (2009). doi:10.1016/j.spl.2009.07.021

9. Kim, Y-H: An estimate on the solutions for stochastic functional differential equations. J Appl Math Informatics. 29(5-5), 1549-1556 (2011)

10. Kim, Y-H: Gronwall, Bellman and Pachpatte type integral inequalities with applications. Nonlinear Anal. 71(12), 2641-2656 (2009). doi:10.1016/j.na.2009.06.009

11. Ren, Y, Xia, N: A note on the existence and uniqueness of the solution to neutral stochastic functional differential equations with infinite delay. Appl Math Comput. 214, 457-461 (2009). doi:10.1016/j.amc.2009.04.013

12. Taniguchi, T: Successive approximations to solutions of stochastic differential equations. J Differ Equ. 96, 152-169 (1992). doi:10.1016/0022-0396(92)90148-G

13. Agarwal, RP, Kim, Y-H, Sen, SK: Multidimensional Gronwall-Bellman-type integral inequalities with applications. Mem Diffe Equ Math Phys. 47, 19-122 (2009)

14. Cho, YJ, Dragomir, SS, Kim, Y-H: On some Gronwall type inequalities involving iterated integrals. Math Inequal Appl. 14(3), 605-620 (2011)

15. Cho, YJ, Dragomir, SS, Kim, Y-H: On some Gronwall type inequalities with iterated integrals. Math Commun. 12(1), 63-73 (2007)

16. Cho, YJ, Kim, Y-H, Pečarić, J: New Gronwall-Ou-lang type integral inequalities and their applications. ANZIAM J. 50(1), 111-127 (2008). doi:10.1017/S1446181108000266

doi:10.1186/1029-242X-2012-126

Cite this article as: Cho et al:: A note on the existence and uniqueness of the solutions to SFDES. Journal of Inequalities and Applications 2012 2012:126.

\section{Submit your manuscript to a SpringerOpen ${ }^{\circ}$} journal and benefit from:

- Convenient online submission

- Rigorous peer review

- Immediate publication on acceptance

- Open access: articles freely available online

- High visibility within the field

- Retaining the copyright to your article

Submit your next manuscript at $\boldsymbol{s p r i n g e r o p e n . c o m ~}$ 